\title{
Study on Transient Negative Sequence Current Calculation and Harmonics Characteristic Analysis for Nuclear Half-Speed Turbine Generator
}

\author{
Dong Chuanyou $^{1,2, *}$, Ge Baojun ${ }^{2}$ and Liang Weiyan ${ }^{1}$ \\ 1. Harbin Institute of Technology, Harbin, China, \\ 2. Harbin University of Science and Technology, Harbin, China \\ dcy131086@163.com
}

\begin{abstract}
In recent years, with the rapid development of China's nuclear power industry, nuclear generator has also been actively developed. Because the unit capacity of the generator is increasing, so working life and operational reliability of the generator are also being tested. Among them, transient thermal running ability of the generator is one of the key factors affecting working life and operational reliability of the generator. This paper mainly analyzed the negative sequence current and the transient negative sequence component of the generator during asymmetric operation, and elaborates the principle of the generator negative sequence component harmonic. The paper has reference value for in-depth study of the generator negative sequence component.
\end{abstract}

Keywords: negative sequence current, transient negative sequence component

\section{Introduction}

At present, in the power structure of our country, thermal power unit still occupies an important position, but according to the economic analysis of Germany's Siemens, the unit cost of 1000MW capacity level units is $30 \%$ lower than the $600 \mathrm{MW}$ capacity level units, Therefore, for the maximizing of economic benefits, nuclear power motor has attracted people's attention [1-2].

With the continuous development of motor manufacturing technology, the unit capacity of motor is increased, the electromagnetic loads and thermal loads of large generators is increased. For the above reasons, the temperature of the various components of the motor is increased, thereby affecting the life of the motor and the operational reliability [3-4]. In recent years, with the development of ventilation and cooling technology, the steady-state thermal operating ability of the large generators has been greatly improved, but the transient-state thermal operating ability have not been improved [5-6]. The temperature and fever which is caused by a variety of conditions negative sequence eddy is one of the important indicators for studying transient thermal generators running ability.

Generator running in asymmetric process will produce negative sequence current, due to the skin effect, the loss of negative sequence current which is produced in the rotor surface is particularly obvious, so the ability to accurately calculate the negative sequence is especially important. In summary, both from theoretical research and practical application, in the running process, the research of electromagnetic and temperature distribution of the steam turbine generator using on nuclear power negative sequence is of great significance. 


\section{The Formation of Negative Sequence Current}

In normal operation, the generator armature windings contain the positive sequence current without negative sequence current and zero-sequence current. When the generator, transformer or transmission line appear failure which resulting in asymmetric operation, the generator armature windings will produce negative sequence current. Negative sequence current and the magnetic field will interact with each other, then it will generate twice power frequency electromagnetic torque, the twice power frequency electromagnetic torque will lead to the stator and rotor of the generator producing mechanical vibration, the twice-frequency is in the natural resonant frequency range of the rotor, so it may cause the rotor resonance, resulting in shaft fatigue wear and aging, the more serious consequences is damage to the motor [7].

Negative sequence magnetic field generated by the current negative sequence will swirl and eddy current losses in the rotor, the eddy current loss can cause the overheating of the rotor, sometimes it may burn the rotor. In the rotor, the heat of structural member are proportional to the time $\mathrm{T}$ and $\left(I_{2}^{*}\right)^{2}$, so for measuring the rotor heating and negative sequence current $I_{2}^{*}$ and the time T relationship, It can be expressed as

$$
\int_{0}^{t} i_{2}^{2} \mathrm{~d} t=\left(I_{2}^{*}\right)^{2} t=\mathrm{A}
$$

\section{The Calculation of Transient Negative Sequence Component}

For decades, the internal fault digital research methods of the stator winding of synchronous generator can be divided into the following categories: symmetrical components, phase coordinate method, multi-loop analysis and finite element method [812]. Symmetrical components which can be see that three-phase current is superimposed by positive sequence components, negative sequence component and zero sequence component. Symmetrical component method is based on the basis of linear superposition, so the nonlinear system cannot be directly applied. This article will use the symmetrical components for the failure analysis of turbo-generator.

\subsection{The Mathematical Model of Symmetrical Components to Calculate the Transient Negative Sequence Component}

When the generator is run in Asymmetric, three-phase unbalanced currents $\dot{I}_{\mathrm{A}}$, $\dot{I}_{\mathrm{B}}, \dot{I}_{\mathrm{C}}$ can be divided into three groups Symmetrical components which are positive sequence current, negative sequence current and zero-sequence current. the following relationship between them:

$$
\begin{aligned}
& \dot{I}_{\mathrm{A}}=\dot{I}_{\mathrm{A}}^{+}+\dot{I}_{\mathrm{A}}^{-}+\dot{I}_{\mathrm{A}}^{0} \\
& \dot{I}_{\mathrm{B}}=\dot{I}_{\mathrm{B}}^{+}+\dot{I}_{\mathrm{B}}^{-}+\dot{I}_{\mathrm{B}}^{0}=a^{2} \dot{I}_{\mathrm{A}}^{+}+a \dot{I}_{\mathrm{A}}^{-}+\dot{I}_{\mathrm{A}}^{0} \\
& \dot{I}_{\mathrm{C}}=\dot{I}_{\mathrm{C}}^{+}+\dot{I}_{\mathrm{C}}^{-}+\dot{I}_{\mathrm{C}}^{0}=a \dot{I}_{\mathrm{A}}^{+}+a^{2} \dot{I}_{\mathrm{A}}^{-}+\dot{I}_{\mathrm{A}}^{0}
\end{aligned}
$$

The character $a$ is a operator, $a=e^{j \frac{2}{3} \pi}$

Symmetrical components of negative-sequence current is expressed as follows:

$$
\dot{I}_{2}=\frac{1}{3}\left(\dot{I}_{\mathrm{A}}+a^{2} \dot{I}_{\mathrm{B}}+a \dot{I}_{\mathrm{C}}\right)
$$


Negative sequence capability of the generator rotor is related with the maximum allowable temperature of the metal surface. The negative sequence capability which is determined by each material component of the maximum allowable temperature is called homeostasis negative sequence ability, the transient negative sequence capability is determined by the transient allowable temperature limits of the rotor surface.

Currently, at home and abroad, the negative sequence current per-unit value $I_{2}{ }^{*}$ is characterize as the negative sequence capability of steady-state generator, the transient negative sequence is described as $\int_{0}^{t} i_{2}^{2} \mathrm{~d} t=\left(I_{2}^{*}\right)^{2} t=A$, where $i_{2}$ is the instantaneous value of the negative sequence current flowing through the generator. The negative sequence capability of the motor is measured through the experimental method, according to the loss relationship that positive and negative sequence current is generated between the rotor surface, the motor which can withstand the maximum negative sequence current is determined.

\subsection{The Negative Sequence Component Analysis of the Short-circuit Fault}

For example, AP1000 type half speed turbine generator, analysis of the motor transient negative sequence component calculation, some parameters of the generator is showed in the following Table:

Table 1. Generator Parameters

\begin{tabular}{c|c|c|c|c|c|c}
\hline $\begin{array}{c}\text { Rated } \\
\text { capacity }\end{array}$ & $\begin{array}{c}\text { Power } \\
\text { factor }\end{array}$ & $\begin{array}{c}\text { Rated } \\
\text { voltage }\end{array}$ & $\begin{array}{c}\text { Rated } \\
\text { current }\end{array}$ & $\begin{array}{c}\text { Excitation } \\
\text { current }\end{array}$ & frequency & speed \\
\hline $1407 \mathrm{MVA}$ & 0.9 & $24 \mathrm{kV}$ & $33847 \mathrm{~A}$ & $9265 \mathrm{~A}$ & $50 \mathrm{~Hz}$ & $1500 \mathrm{r} / \mathrm{min}$ \\
\hline
\end{tabular}

Setting the initial state of the generator is load operation, three-phase short circuit is occurred suddenly in the initial phase angle $\alpha_{0}=0^{\circ}$ (A vertical axis relative to the axis of the straight), this time the three-phase current is as followed:

$$
\left\{\begin{array}{l}
i_{\mathrm{A}}=-\left[\left(I_{\mathrm{m}}^{\prime \prime}-I_{\mathrm{m}}^{\prime}\right) e^{-\frac{t}{T_{\mathrm{d}}^{\prime \prime}}}+\left(I_{\mathrm{m}}^{\prime}-I_{\mathrm{m}}\right) e^{-\frac{t}{T_{\mathrm{d}}^{\prime}}}+I_{\mathrm{m}}\right] \cos \left(\omega t+\alpha_{0}\right)+I_{\mathrm{m}}^{\prime \prime} \cos \alpha_{0} e^{-\frac{t}{T_{\mathrm{a}}}} \\
i_{\mathrm{B}}=-\left[\left(I_{\mathrm{m}}^{\prime \prime}-I_{\mathrm{m}}^{\prime}\right) e^{-\frac{t}{T_{\mathrm{d}}^{\prime \prime}}}+\left(I_{\mathrm{m}}^{\prime}-I_{\mathrm{m}}\right) e^{-\frac{t}{T_{\mathrm{d}}^{\prime}}}+I_{\mathrm{m}}\right] \cos \left(\omega t+\alpha_{0}-120^{\circ}\right)+I_{\mathrm{m}}^{\prime \prime} \cos \left(\alpha_{0}-120^{\circ}\right) e^{-\frac{t}{T_{\mathrm{a}}}} \\
i_{\mathrm{C}}=-\left[\left(I_{\mathrm{m}}^{\prime \prime}-I_{\mathrm{m}}^{\prime}\right) e^{-\frac{t}{T_{\mathrm{d}}^{\prime \prime}}}+\left(I_{\mathrm{m}}^{\prime}-I_{\mathrm{m}}\right) e^{-\frac{t}{T_{\mathrm{d}}^{\prime}}}+I_{\mathrm{m}}\right] \cos \left(\omega t+\alpha_{0}-240^{\circ}\right)+I_{\mathrm{m}}^{\prime \prime} \cos \left(\alpha_{0}-240^{\circ}\right) e^{-\frac{t}{T_{\mathrm{a}}}}
\end{array}\right.
$$

The non-periodic component of the A phase short-circuit current is:

$$
i_{\mathrm{A}=}=I_{\mathrm{m}}^{\prime \prime} \cos \alpha_{0} e^{-\frac{t}{T_{\mathrm{a}}}}
$$

The three-phase stator windings in spatial arrangement is $120^{\circ} / p$ between each other, $p$ is the pole pairs, for 4-pole motor, three-phase non-periodic component in the spatial arrangement is $60^{\circ} 120^{\circ} / p$ between each other, which is formed of a resultant magnetic field, the non-periodic component of the phase is said as $i_{r}$ :

$$
i_{\mathrm{r}}=\frac{1}{2} I_{\mathrm{m}}^{\prime \prime} \cos \alpha_{0} e^{-\frac{t}{T_{\mathrm{a}}}}, \quad I_{\mathrm{m}}^{\prime \prime}=\frac{\sqrt{2}}{X_{\mathrm{d}}^{\prime \prime}} E_{0}
$$


From Equation $8, i_{\mathrm{r}}$ shows the amplitude, when $\alpha_{0}=0^{\circ}$ and $180^{\circ}, i_{r}$ is maximum, when $\alpha_{0}=90^{\circ}$ and $270^{\circ}$ is minimum, Figure 1 is the synthesized curves when the threephase current $i_{r}$ is $\alpha_{0}=0^{\circ}, 30^{\circ}$ and $60^{\circ}$.

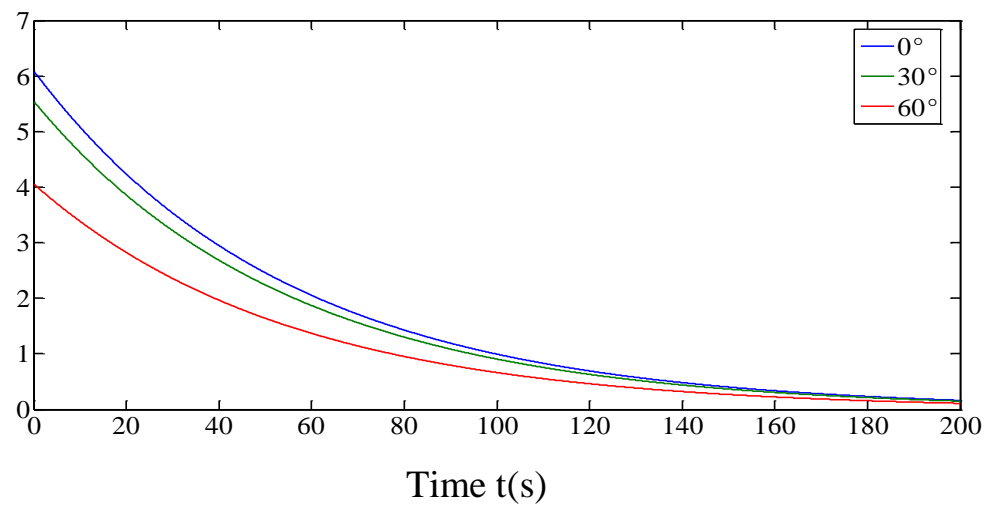

Figure 1. The Synthetic Current of Non-periodic Component

Transient negative sequence ability is calculated based on $\int_{0}^{t} i_{2}^{2} \mathrm{~d} t=\left(I_{2}^{*}\right)^{2} t=A$, so here using the square with a synthetic non-periodic component of the current to the integral of time represent the non-periodic component equivalent negative sequence ,because the stator current loss in the surface of the stator is proportional to the square of the frequency, imputed to the negative sequence current under the same frequency so that the frequency is multiplied by the imputed coefficient $1 / 4, A_{2}$ the equivalent negative sequence of synthetic non-periodic component is indicated by $A_{2}$ :

$$
A_{2}=\frac{1}{4} \int_{0}^{t} i_{\mathrm{r}}^{2} \mathrm{~d} t=\frac{1}{16}\left(\cos ^{2} \alpha_{0}\right) I_{\mathrm{m}}^{\prime \prime 2} \frac{T_{\mathrm{a}}}{2}\left(1-e^{-\frac{2 t}{T_{\mathrm{a}}}}\right)
$$

when $t=60 \mathrm{~s}$, the value of equation $\int_{0}^{t} i_{2}^{2} \mathrm{~d} t=\left(I_{2}^{*}\right)^{2} t=A$ is calculated, at this time, this value is represented as the transient negative sequence ability. So among the transient negative sequence generator ability $\mathrm{A}$, in addition to containing the losses which is generated by the stator negative sequence current it also contains the losses which is generated by non-periodic component ,it is composed by $A_{1}$ and $A_{2}$.

$$
A=A_{1}+A_{2}
$$

According to the design standards of the motor, requiring them to be able to withstand the transient negative sequence ability $\mathrm{A}=5 \mathrm{~s}$. The losses $A_{1}$ which is generated by the negative sequence current in the rotor is independent of the initial angle. The losses from non-periodic component, when the $\alpha_{0}=0^{\circ}, A_{2}$ is the maximum, when $\alpha_{0}=90^{\circ}, A_{2}$ is the 0 s.

(1) When the generator phase short-circuit, due to symmetrical three-phase current, no negative sequence currents, so $=0 \mathrm{~s}$. Calculated, when the worst case, only the nonperiodic component losses on the rotor at about $\mathrm{t}=1 \mathrm{~s}$ maximum $2.66 \mathrm{~s}$. 


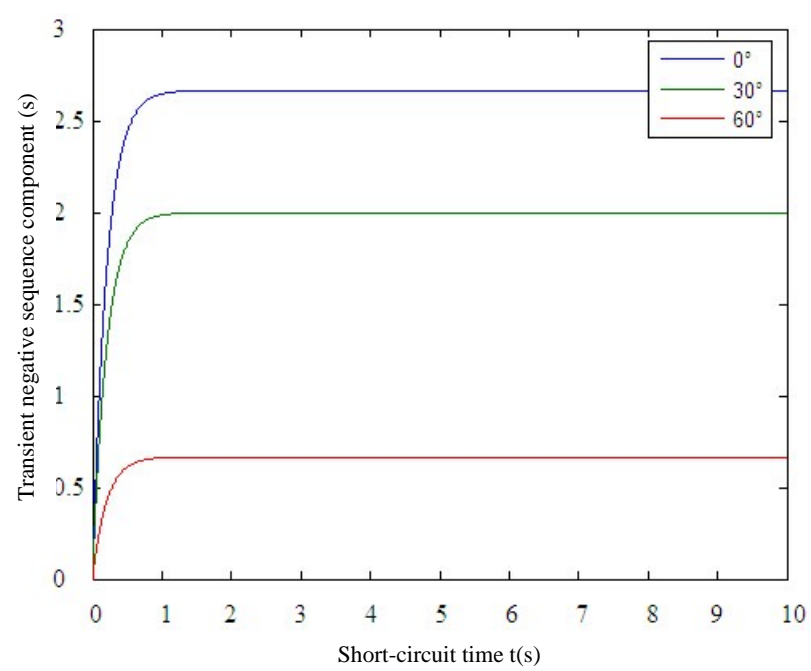

\section{Figure 2. The Equivalent Transient Negative Sequence of Non-periodic} Component

From the above calculation and analysis, within the scope $\alpha_{0}=0^{\circ} \sim 90^{\circ}$, the impact of the non-periodic component to the transient negative sequence shows a decreasing trend, when $\alpha_{0}=0^{\circ}$, the impact is serious..

(2) When the generator occurs an external phase short circuit, when $\alpha_{0}=90^{\circ}, A_{2}=0 \mathrm{~s}$ , this time to calculate the transient $\mathrm{A}$ which is generated by negative sequence, when transient negative $A=5 \mathrm{~s}$, computing $\mathrm{t}=22.5 \mathrm{~s}$. setting the short initial angles $\alpha_{0}$ as $0^{\circ}$ 、 $30^{\circ} 、 60^{\circ}$, the value of $\mathrm{t}$ respectively is $13.46 \mathrm{~s}, 15.72 \mathrm{~s}, 20.24 \mathrm{~s}$. the per unit of negative sequence current is $I_{2}{ }^{*}=0.67$.

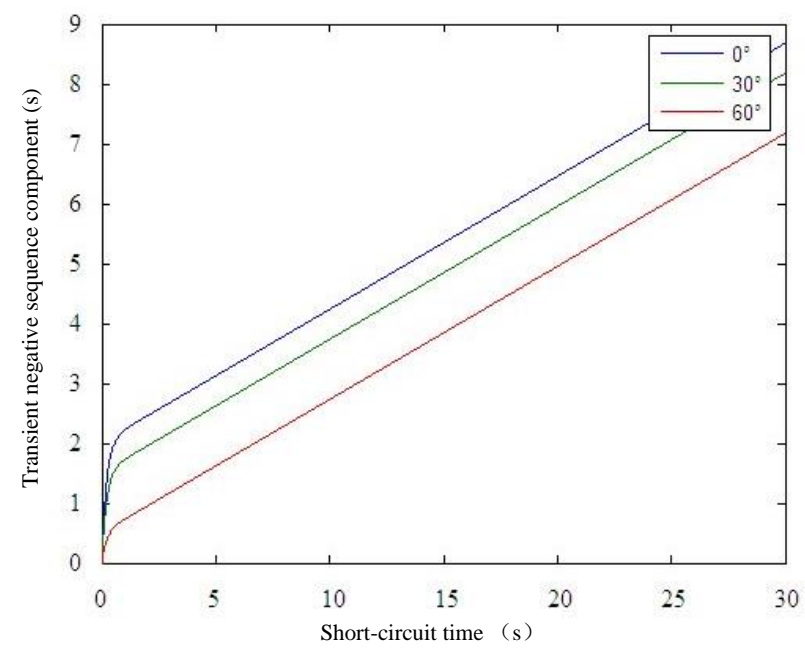

Figure 3. The Transient Negative Sequence of Interphase Short Circuit

In this case the calculation of transient negative sequence if using traditional algorithms to calculate the transient negative sequence, only to calculate the negative sequence current is $t=22.5 \mathrm{~s}$, while using the formula (9) and (10), the value of $t$ should be between $13.46 \sim 22.5 \mathrm{~s}$, this suggests that transient negative sequence may not accurately indicate the actual ability of the damage. 


\section{Harmonic Generation Mechanism of Asymmetric Load Conditions}

\subsection{The Mechanism of Negative Sequence Component Leading the Turbogenerator Generated Harmonics}

When the synchronous generator is in asymmetric conditions, in the armature windings, the fundamental frequency $\mathrm{AC}$ quantities is generated. This fundamental frequency can be divided into positive, negative and zero-sequence component, the magnetic field which is in the air gap is generated by the positive sequence component; in the three-phase winding, the pulsating magnetic field which is generated by the zero sequence component of the fundamental frequency pulsating magnetic field is the same size and the same phase.

Since the three-phase stator windings is spatially symmetric, zero-sequence field is formed for each phase winding of the leakage magnetic field, but impossible to form a composite magnetic field in the rotor space, and thus has no effect on the rotor winding.

$$
\left[\begin{array}{l}
\dot{I}_{+} \\
\dot{I}_{-} \\
\dot{I}_{0}
\end{array}\right]=\frac{1}{3}\left[\begin{array}{lll}
1 & a & a^{2} \\
1 & a^{2} & a \\
1 & 1 & 1
\end{array}\right]\left[\begin{array}{l}
\dot{I}_{\mathrm{A}} \\
\dot{I}_{\mathrm{B}} \\
\dot{I}_{\mathrm{C}}
\end{array}\right]
$$

\subsection{Harmonic Analysis Principle}

In the international, Harmonic is defined as one cycle sine wave components of the electrical component, whose frequency is an integer multiple of the fundamental frequency.

Fourier series decomposition method is an effective way to study harmonic. as long as the arbitrary waveform meet the Dilikeli, then the conditions can be broken down into the following Fourier series:

$$
f(t)=A_{0}+\sum_{h=1}^{\infty}\left(A_{\mathrm{h}} \cos \left(h \omega_{0} t\right)+B_{h} \sin \left(h \omega_{0} t\right)\right)=A_{0}+\sum_{h=1}^{\infty} C_{\mathrm{h}} \sin \left(h \omega_{0} t+\phi_{\mathrm{h}}\right)
$$

$f(t)$ - the periodic function, frequency is $f_{0}$, the angular frequency $\omega_{0}=2 \pi f_{0}$, period $\mathrm{T}=1 / f_{0}=2 \pi / \omega_{0}$;

$C_{1} \sin \left(h \omega_{0} t+\phi_{1}\right)-h$ fundamental component;

$C_{\mathrm{h}} \sin \left(h \omega_{0} t+\phi_{\mathrm{h}}\right)-h$ harmonic componen, Amplitude $C_{\mathrm{h}}$, frequency $h f_{0}$, phase $\phi_{h}$ 。

The coefficients of Fourier series is as followed.

$$
\begin{gathered}
A_{0}=\frac{1}{\mathrm{~T}} \int_{0}^{\mathrm{T}} f(t) \mathrm{d} t=\frac{1}{2 \pi} \int_{0}^{2 \pi} f(t) \mathrm{d} x\left(x=\omega_{0} t\right) \\
A_{\mathrm{h}}=\frac{2}{\mathrm{~T}} \int_{0}^{\mathrm{T}} f(t) \cos \left(h \omega_{0} t\right) \mathrm{d} t=\frac{1}{\pi} \int_{0}^{2 \pi} f(t) \cos (h x) \mathrm{d} x \\
B_{\mathrm{h}}=\frac{2}{\mathrm{~T}} \int_{0}^{\mathrm{T}} f(t) \sin \left(h \omega_{0} t\right) \mathrm{d} t=\frac{1}{\pi} \int_{0}^{2 \pi} f(t) \sin (h x) \mathrm{d} x \\
C_{\mathrm{h}}=\sqrt{A_{\mathrm{h}}^{2}+B_{\mathrm{h}}^{2}} \\
\phi_{\mathrm{h}}=\arctan \left(\frac{A_{\mathrm{h}}}{B_{\mathrm{h}}}\right)
\end{gathered}
$$




\section{(1) Symmetry}

The characteristic of the odd symmetry function is $f(-t)=-f(t)$, when the function is expanded into a Fourier series, it contain sine but no cosine; The characteristic of the even symmetric function is $f(-t)=f(t)$, when the function is expanded into a Fourier series ,it contain no sine; The characteristic of half-wave symmetry is $f\left(t \pm \frac{\mathrm{T}}{2}\right)=-f(t)$, when the function is expanded into a Fourier series ,it contain no DC component and even harmonics; The composition of the power system are generally two-way symmetry element, the voltage and current which is generated by these elements have a half-wave symmetry. This feature allows that the power system harmonic analysis can ignore the even harmonics.

\section{(2) The Measurement Method of Harmonic Distortions}

Based on the theory of Fourier series described earlier, the periodic current or voltage waveform distortion is expanded into Fourier series:

$$
\begin{aligned}
& i(t)=\sum_{\mathrm{h}=1}^{\mathrm{H}} i_{\mathrm{h}}(t)=\sum_{\mathrm{h}=1}^{\mathrm{H}} \sqrt{2} I_{\mathrm{h}} \cos \left(h \omega_{0} t+\theta_{\mathrm{h}}\right) \\
& u(t)=\sum_{\mathrm{h}=1}^{\mathrm{H}} u_{\mathrm{h}}(t)=\sum_{\mathrm{h}=1}^{\mathrm{H}} \sqrt{2} U_{\mathrm{h}} \cos \left(h \omega_{0} t+\varphi_{\mathrm{h}}\right)
\end{aligned}
$$

where:

$I_{\mathrm{h}}-h$ harmonic current valid values .

$\theta_{\mathrm{h}}-h$ harmonic current phase;

$U_{\mathrm{h}}-h$ harmonic voltage valid values;

$\varphi_{\mathrm{h}}-h$ harmonic voltage phase;

$\omega_{0}$-Fundamental angular frequency ; $\omega_{0}=2 \pi f_{0}$;

$f_{0}$-Fundamental frequency, $f_{0}=50 \mathrm{~Hz}$;

$\mathrm{H}$ - the order of high harmonics。

the valid values of distortion voltage and current [14]:

$$
\begin{aligned}
& U_{\mathrm{RMS}}=\sqrt{\frac{1}{\mathrm{~T}} \int_{0}^{\mathrm{T}} u^{2}(t) \mathrm{d} t}=\sqrt{\sum_{\mathrm{h}=1}^{\mathrm{H}} U_{\mathrm{h}}^{2}} \\
& I_{\mathrm{RMS}}=\sqrt{\frac{1}{\mathrm{~T}} \int_{0}^{\mathrm{T}} i^{2}(t) \mathrm{d} t}=\sqrt{\sum_{\mathrm{h}=1}^{\mathrm{H}} I_{\mathrm{h}}^{2}}
\end{aligned}
$$

Voltage distortion factor VDF, also known as voltage total harmonic distortion $\mathrm{THD}_{\mathrm{V}}$, current distortion factor CDF, also known as the current total harmonic distortion $\mathrm{THD}_{\mathrm{I}}$ :

$$
\begin{aligned}
& \operatorname{THD}_{\mathrm{U}}=\frac{1}{U_{1}} \sqrt{\sum_{\mathrm{h}=2}^{\mathrm{H}} U_{\mathrm{h}}^{2}}=\sqrt{\left(\frac{U_{\mathrm{RMS}}}{U_{1 \mathrm{RMS}}}\right)^{2}-1} \\
& \mathrm{THD}_{\mathrm{I}}=\frac{1}{I_{1}} \sqrt{\sum_{\mathrm{h}=2}^{\mathrm{H}} I_{\mathrm{h}}^{2}}=\sqrt{\left(\frac{I_{\mathrm{RMS}}}{I_{1 \mathrm{RMS}}}\right)^{2}-1}
\end{aligned}
$$

where: 
$U_{1}$-Fundamental voltage amplitude ;

$I_{1}$-Fundamental current amplitude 。

\section{Conclusion}

(1) in the transient negative sequence ability A, it not only contain the losses which is generated by the negative sequence current ,but also contain the losses which is generated by Non-periodic component of the current.

(2)At the interaction between rotating magnetic field in the stator and pulsating magnetic field in the rotor winding currents, stator current contains odd harmonics, while the rotor current contain an even number harmonic components;

(3) The power system generally have a two-way, so in order to analyze the power system harmonic, it can ignore the even harmonics.

\section{References}

[1] Y. Guo, "Power system reliability analysis [M]", Beijing: Tsinghua University Press, (2003), pp. 14-19.

[2] S. Bo, "Negative sequence current generator rotor hazard analysis and preventive measures [J]", Zhejiang Electric Power, vol. 5, (2007), pp. 35-38.

[3] G. Mao, "Our large turbo accidents and quality problems [J]", Grid technology, vol. 24, no. 11, (2000), pp. 1-6.

[4] Von rebirth, "In recent years, large turbo cause of the accident and preventive measures [J]", Grid technology, vol. 23, no. 1, (1999), pp. 74-78.

[5] Shi-yuan and S. Huang, "AC motor winding theory [M]", Beijing: China Electric Power Press, (2007), pp. 1-13.

[6] X. Hu and C. P. Zhang, "The finite element calculation of Turbo generator rotor negative sequence eddy field [J]”, Tsinghua University (Natural Science), vol. 01, (1979), pp. 60-71.

[7] A. G. Jack, "Negative-sequence Currents and Losses in The Solid Rotor of a Turbo-generator [J]", Generation, Transmission and Distribution, IEE Proceedings C, vol. 127, no. 2, (1980), pp. 53-64.

[8] S. J. Salon, M. R. Shah and L. W. Montgomery, "Analysis and Testing of Negative Sequence Heating of Turbine Generators Rotors [J]”, IEEE Transactions on Power Apparatus and Systems, vol. 100, no. 8, (1981), pp. 3940-3949.

[9] T. Bach, D. Wohner and K. Takahashi, "Determining Negative Sequence Currents of Turbine Generator Rotors [C]", Electrical Machines and Systems, Tokyo, Japan, (2009), pp. 1-6.

[10] J. P. Sturgess, P. S. Sangha and T. W. Preston, "Comparison of Finite Element Methods for Turbine Generator Negative Sequence Performance Pre-diction [C]”, Electrical Machines and Drives. Fourth International Conference, London, England: (1989), pp. 106-110.

[11] N. A. Demerdash and H. B. Hamilton, "Simulation for Design Purposes of Magnetic Fields in Turbogenerators with Symmetrical and Asymmetrical Rotors [J]", IEEE Transactions on Power Apparatus and Systems, vol. 91, no. 5, (1972), pp. 1985-1999. 